cover whether individuals spend much of their time hidden in the mud safe from the fishing nets. They dived in the relatively shallow water of Loch Torridon, on the west coast of Scotland, to count Nephrops and its burrows, and tracked animals moving out of their burrows by recording their footprints on small trays of 'Vaseline' placed outside the entrances. The form of some burrows was investigated by the preparation of casts from polyester resins. This technique was carried out with considerable success, and Rice had several casts, about one metre across, with him at the meeting.

In 1969 the density of burrows in ninety square metres of mud was recorded as $1 / 1.4 \mathrm{~m}^{2}$, and there was one Nephrops in every $5.5 \mathrm{~m}^{2}$. The animals, apparently, had no permanent homes, sometimes moving rapidly from burrow to burrow and sometimes staying in one place for a longer time. It was clear that in the relatively shallow water of Loch Torridon, Nephrops tends to stay hidden away during the day, coming out during the night. This is reminiscent of trawl catches which are generally greater during the night in shallow water.

The situation was quite different in the deeper water of the Sound of Jura, also on the west coast of Scotland. Chapman's data, collected with an underwater television camera towed under a research vessel, show that Nephrops was much more active during the day than during the night. Rice thinks that light intensity is the cause of this difference in burrowing behaviour. He suggested that Nephrops has an optimum range in which it can be active, so that in deep water light intensity is too low at night and in shallow water it is too high during the day.

If this seems to have solved one problem, there is still the mystery of the apparent absence of young Nephrops. Although in three years Rice and Chapman have seen only adults, with a carapace more than $30 \mathrm{~mm}$ long, the young ones must be somewhere in both Loch Torridon and the Sound of Jura. They hope to find them yet.

\section{SEMICONDUCTORS \\ Evidence for Bonding}

from our Solid State Physics Correspondent

AN upsurge of interest in the chemical or bonding characteristics of semiconductors has followed the work of J. C. Phillips (Phys. Rev. Lett., 20, 550; 1969) and others in which the key to a better understanding of the chemical and physical properties of semiconductors and insulators was held to lie in a detailed knowledge of the distribution of the electronic charge inside the solid. Accurate calculations of band structure have already been carried out but little effort has been made to reproduce the electron charge density in these materials to an equivalent accuracy.

J. P. Walter and M. L. Cohen of the University of California at Berkeley (Phys. Rev. Lett., 26, 17; 1971) have now evaluated the charge density contours for the valence electrons in germanium, gallium arsenide and four other crystalline materials and have discovered that the nature of the bonding is clearly portrayed by these contours. More significantly, the calculations have provided direct support for the notion that structures of four-fold coordination with covalent bonding can be differentiated neatly from six-fold structures held together by electrostatic forces by the value of the ionicity parameter as defined by Phillips and Van Vechten.
This idea had been arrived at previously only on empirical grounds.

Walter and Cohen computed the electronic charge from wave functions derived in calculations of pseudopotential band structure. Up to ninety plane waves were needed to represent the wave functions. Several striking features appear in the contours of charge density : for germanium, for example, the tetrahedral covalent bonding can be seen clearly from the concentration of electronic charge halfway between adjacent atoms. The greater ionic character of GaAs can be detected from the shift of the centre of the bonding charge away from the halfway position towards the As atoms. The total amount of bonding charge also gets less in going from Ge to

\title{
Unusual Ribosomes of Mitochondria
}

Mitochondria possess both their own genetic apparatus and the machinery of protein biosynthesis, and there is much current interest in the characteristics of these structures within these little organelles. This is reflected by the appearance of a batch of three articles in next Wednesday's Nature New Biology dealing with mitochondrial ribosomes.

Brega and Vesco and Attardi and Ojala have studied the structures involved in the biosynthesis of proteins within mitochondria from the human cell line HeLa, and have found three types of ribonucleoprotein particles present within the mitochondria, with sedimentation coefficients of $55-60 \mathrm{~S}, 40-45 \mathrm{~S}$ and $30-35 \mathrm{~S}$. These workers prove beyond doubt that the heaviest particles are single ribosomes, and that the two lighter classes are the two types of ribosomal sub-particles, containing RNAs of $16 \mathrm{~S}$ and $12 \mathrm{~S}$ size. Both the ribosomes and their constituent RNAs are, of course, remarkably small compared with cytoplasmic ribosomal particles of either eukaryotic or prokaryotic cells, and similar findings have already been reported for ribosomes from the mitochondria of other types of animal cells.

The structures active in protein synthesis within intact mitochondria have been found to be polysomes containing several 55-60S ribosomes, and Brega and Vesco have isolated polysomes with sedimentation coefficients as large as $200 S$. The considerable technical difficulties involved in demonstrating this were overcome by inhibiting cytoplasmic protein synthesis specifically with the cytotoxic drugs pederine or cycloheximide, which do not interfere with mitochondrial protein synthesis. Proteins synthesized in the presence of these drugs were labelled by using radioactive amino-acids as precursors. Labelled polysomes were then obtained from isolated mitochondria by dissolving them with detergent. But addition of chloramphenicol, which is known to specifically block mitochondrial protein synthesis, prevented the formation of labelled polysomes. Puromycin causes the disappearance of radioactivity from both the monosome and polysome fractions, presumably by releasing the nascent polypeptide chains.

Attardi and Ojala present an interesting speculation about the evolution of mitochondrial ribosomal RNA. They point out that the ribosomal RNAs of animal cell mitochondria are considerably smaller than those of mitochondria from lower eukaryotic cells, and that a parallel decrease in the size of mitochondrial DNA has also occurred. The mitochondrial DNA of animal cells is usually about $5 \mu$ in length, and they suggest that this is a lower size limit, representing the minimum amount of information necessary for mitochondrial function. They postulate that the reduction in size of the ribosomal cistrons was a part of the overall diminution of the mitochondrial genome during evolution.

In a third article, Lizardi and Luck reveal that mitochondrial ribosomes from Neurospora lack a 5S RNA, previously thought to be a universal constituent of ribosomes. They took the essential precaution of preparing material entirely uncontaminated by cytoplasmic ribosomes, by isolating the mitochondria by isopycnic centrifugation through sucrose gradients. Electrophoresis of RNA from whole mitochondria, or from extracted ribosomes and polysomes, shows that no 7S or 5S RNA components are present and Lizardi and Luck thus rule out that the RNA is likely to be leached out of the ribosomes in the course of extraction. Even more conclusively, they find that labelled RNA synthesized by isolated mitochondria does not contain either of these components (although short pulses yield 5S-like material which they postulate to be tRNA precursor molecules). 